\title{
Breast cancer risk factors, survival and recurrence, and tumor molecular subtype: analysis of 3012 women from an indigenous Asian population
}

Mustapha Abubakar ${ }^{1}$, Hyuna Sung ${ }^{1,2}$, Devi BCR ${ }^{3}$, Jennifer Guida ${ }^{4}$, Tieng Swee Tang ${ }^{3}$, Ruth M. Pfeiffer ${ }^{1}$ and Xiaohong R. Yang ${ }^{1 *}$ (D)

\begin{abstract}
Background: Limited evidence, mostly from studies in Western populations, suggests that the prognostic effects of lifestyle-related risk factors may be molecular subtype-dependent. Here, we examined whether pre-diagnostic lifestyle-related risk factors for breast cancer are associated with clinical outcomes by molecular subtype among patients from an understudied Asian population.

Methods: In this population-based case series, we evaluated breast cancer risk factors in relation to 10-year all-cause mortality (ACM) and 5-year recurrence by molecular subtype among 3012 women with invasive breast cancer in Sarawak, Malaysia. A total of 579 deaths and 314 recurrence events occurred during a median follow-up period of $\sim 24$ months. Subtypes (luminal A-like, luminal B-like, HER2-enriched, triple-negative) were defined using immunohistochemical markers for hormone receptors and human epidermal growth factor receptor 2 (HER2) in conjunction with histologic grade. Hazard ratios (HRs) and 95\% confidence intervals (Cls) for the associations between risk factors and ACM/recurrence were estimated in subtype-specific Cox regression models.

Results: We observed heterogeneity in the relationships between parity/breastfeeding, age at first full-term pregnancy (FFP), family history, body mass index (BMI), and tumor subtype ( $p$ value $<0.05)$. Among luminal A-like patients only, older age at menarche $[\mathrm{HR}(95 \% \mathrm{Cl}) \geq 15$ vs $\leq 12$ years $=2.28(1.05,4.95)]$ and being underweight $\left[\mathrm{HR}_{\mathrm{BMI}}<\right.$

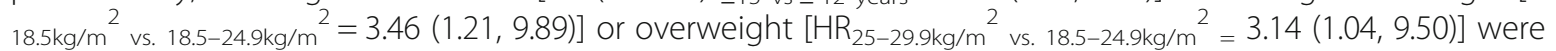
associated with adverse prognosis, while parity/breastfeeding $\left[\mathrm{HR}_{\text {breastfeeding vs nulliparity }}=0.48(0.27,0.85)\right]$ and older age at FFP $[H R>30$ vs $<21$ years $=0.20(0.04,0.90)]$ were associated with good prognosis. For these women, the addition of age at menarche, parity/breastfeeding, and BMI, provided significantly better fit to a prognostic model containing standard clinicopathological factors alone $\left[\operatorname{LRX}^{2}(8 d f)=21.78 ; p\right.$ value $\left.=0.005\right]$. Overall, the results were similar in relation to recurrence.

Conclusions: Our finding that breastfeeding and BMI were associated with prognosis only among women with luminal A-like breast cancer is consistent with those from previously published data in Western populations. Further prospective studies will be needed to clarify the role of lifestyle modification, especially changes in BMI, in improving clinical outcomes for women with luminal A-like breast cancer.
\end{abstract}

Keywords: Breast cancer, Risk factors, Survival, Recurrence, Molecular subtype

\footnotetext{
* Correspondence: royang@mail.nih.gov

${ }^{1}$ Integrative Tumor Epidemiology Branch, Division of Cancer Epidemiology and Genetics, National Cancer Institute (NCl), National Institutes of Health, 9609 Medical Center Drive, Rockville, MD 20850, USA

Full list of author information is available at the end of the article
}

(c) The Author(s). 2018 Open Access This article is distributed under the terms of the Creative Commons Attribution 4.0 International License (http://creativecommons.org/licenses/by/4.0/), which permits unrestricted use, distribution, and reproduction in any medium, provided you give appropriate credit to the original author(s) and the source, provide a link to the Creative Commons license, and indicate if changes were made. The Creative Commons Public Domain Dedication waiver (http://creativecommons.org/publicdomain/zero/1.0/) applies to the data made available in this article, unless otherwise stated. 


\section{Background}

In addition to impacting incidence, lifestyle and environmental risk factors for breast cancer may influence disease progression. Several studies have previously evaluated this question, with mixed results. While some studies have documented older age at menarche [1-3], early age at first full-term pregnancy (FFP) $[4,5]$ and nulliparity $[6,7]$ to be associated with adverse prognosis in breast cancer patients, others have reported better prognosis in relation to these risk factors [8-12]. Discrepancies in reported associations may be explained by differences in study populations, risk factor distributions, and potential confounders, but could also be due to heterogeneity inherent in breast cancer.

Findings from expression profiling studies have been used to classify breast cancers into intrinsic subtypes (i.e. luminal A, luminal B, human epidermal growth factor receptor 2 (HER2)-enriched, basal-like, and normal-like subtypes), which were associated with different prognoses [13] and can be corroborated by immunohistochemical (IHC) markers for hormone receptors (i.e. estrogen receptor (ER), progesterone receptor [PR]) and HER2. Recently, proxies of the extent of tumor proliferation have been endorsed to refine subgroups that recapitulate the intrinsic subtypes more accurately than using hormone receptors and HER2 alone [14, 15]. Epidemiological studies have shown that associations between breast cancer risk factors vary by tumor subtypes. For example, parity and early age at FFP are associated with decreased risk of luminal breast tumors, but they do not protect and may even increase the risk for ER-negative or triple-negative breast cancers [16-19].

Three previous studies have evaluated the relationship between breast cancer risk factors and survival according to molecular subtype, one among women in Seoul, South Korea [20] and the other two involving analyses of US-based prospective breast cancer cohorts [21, 22]. Results from these studies suggest that the associations between late age at menarche [20], breastfeeding [21], high body mass index (BMI) [22] and survival after breast cancer might differ according to molecular subtype. However, findings from these studies are yet to be validated in independent populations and, to our knowledge, no study has specifically examined risk factors in relation to survival according to subtypes defined by the recent IHC classification scheme accounting for proliferation in an Asian population.

Despite racial and geographic variations in the incidence, presentation, and outcome of breast cancer; so far, most investigations on risk factors in relation to tumor subtypes and survival have been conducted in European populations. This analysis, therefore, aims to evaluate the association between breast cancer risk factors and tumor molecular subtypes, defined by hormone receptors and HER2 in conjunction with histologic grade; and to examine the relationship between risk factors and survival by molecular subtype among women in Sarawak, Malaysia.

\section{Methods \\ Study population}

Sarawak is a Malaysian state on Borneo with a multiethnic composition, comprising of native Borneo populations (51\%), Chinese (25\%) and Malays (24\%) [23]. Overall, 3355 women with invasive breast cancer diagnosed and treated between 2003 and 2016 in the Department of Radiotherapy, Oncology, and Palliative Care, Sarawak General Hospital where 93\% of all breast cancer cases diagnosed in Sarawak are treated, were recruited for this study. Of these, $106(\sim 3 \%)$ did not participate by not filling the questionnaire leading to a participating rate of $\sim 97 \%$. Of the 3249 who participated, $168(\sim 5 \%)$ were lost to follow-up and 69 did not have complete information on ER, PR, HER2, and grade that is needed to generate breast cancer subtypes hence were excluded from further analysis. Ultimately, 3012 women representing $\sim 90 \%$ of the original population were included in the current analysis. Information on lifestyle and environmental risk factors were obtained from questionnaires that were administered to participants at enrollment, which was approximately 4 weeks after diagnosis, while information on tumor characteristics was obtained from clinical records. Weight and height measurements were obtained in the clinic as part of the clinical workup for the calculation of chemotherapy doses. Recordings were performed by a trained member of staff using a weighing scale. Patients were given follow-up appointments to the clinic during which recurrence was evaluated and clinically confirmed. For those living in the outskirts of the city, if recurrence was suspected, the patients were referred to our clinic for further evaluation. Furthermore, a research assistant made regular calls to check the patient's status, whether alive or dead. The current analysis included a follow-up period of 153 months (median follow-up = 24 months). Ethical approval for this project was provided by the Ethics Committee of the National Institutes of Health, Malaysia. This study did not involve the use of personal identifying information; hence, it was exempted from review by the National Institutes of Health (NIH) Office of Human Subject Research Protections [23].

\section{Breast cancer subtype definition}

IHC staining for ER, PR, and HER2 was performed on formalin-fixed, paraffin-embedded tissue sections as has been previously described [24]. Molecular subtypes were defined using the St Gallen classification, proposed for the recapitulation of the intrinsic subtypes using IHC 
and proliferation markers $[14,15]$. According to the St Gallen classification scheme, luminal breast cancers can be further distinguished into subgroups based on their level of proliferation (using KI67 or histologic grade) and hormone receptor expression patterns. Accordingly, luminal tumors that homogeneously express hormone receptors (i.e. $\mathrm{ER}+$ and $\mathrm{PR}+$ ) and low proliferation are classified as luminal A-like while those that heterogeneously express hormone receptors (i.e. $\mathrm{ER}^{+} / \mathrm{PR}^{-}$or $\mathrm{ER}^{-} / \mathrm{PR}^{+}$) and/or those that homogeneously or heterogeneously express hormone receptors (i.e. ER+ and/or PR +) but are also high proliferating (high KI67 or grade 3) and/or HER2+ are classified as luminal B. In keeping with this definition, we utilized ER, PR, and HER2 in addition to histologic grade [25], to define subtypes as follows: Luminal A-like: $\mathrm{ER}^{+}$and $\mathrm{PR}^{+}, \mathrm{HER}^{-}$and low grade (histologic grade 1 or 2); Luminal B-like: $\mathrm{ER}^{+}$and/ or $\mathrm{PR}^{+}, \mathrm{HER} 2^{-}$and high-grade (histologic grade 3 ) or $\mathrm{ER}^{+}$and/or $\mathrm{PR}^{+}, \mathrm{HER}^{+}$(regardless of levels of histologic grade); HER2-enriched: $\mathrm{ER}^{-}$and $\mathrm{PR}^{-}$and $\mathrm{HER}^{+}$; and triple-negative: $\mathrm{ER}^{-}$and $\mathrm{PR}^{-}$and $\mathrm{HER} 2^{-}$.

\section{Statistical analysis}

Frequency tables were used to assess the distribution of risk factors and clinicopathological characteristics among the different subtypes. The chi-square test was used to assess differences for categorical variables and the Kruskal-Wallis test was used for continuous variables.

We categorized risk factors based on what is the convention for each variable and in accordance with what has been published in large-scale studies of breast cancer $[18,26]$. We categorized age at menarche $[\leq 12$ years (early menarche), 13, 14 and $\geq 15$ years (late menarche)]; family history of breast cancer in a first-degree relative [yes and no]; age at FFP $[<21,21-24.9,25-30,>30$ years] and age at menopause $[\leq 50$ and $>50$ years] similarly as in previously published articles [18, 26]. For BMI, we adopted the World Health Organization classification $\left[<18.5 \mathrm{~kg} / \mathrm{m}^{2}\right.$ (underweight); $18.5-24.9 \mathrm{~kg} / \mathrm{m}^{2}$ (normal weight); $25-29.9 \mathrm{~kg} / \mathrm{m}^{2}$ (overweight) and $\geq 30 \mathrm{~kg} / \mathrm{m}^{2}$ (obese)]. To test for associations between risk factors and molecular subtypes, we constructed a polytomous logistic regression model with tumor subtype as the outcome (luminal A-like subtype as the reference category) and risk factors (age at menarche $[\leq 12$ years (early menarche, reference category), 13, 14, and $\geq 15$ years (late menarche)], parity and breastfeeding [nulliparity (reference category), parity but no breastfeeding, parity and breastfeeding], age at FFP $[<21$ (reference category), 21-24.9, 25-30, > 30 years], family history [yes and no (reference category)], and BMI $\left[<18.5 \mathrm{~kg} / \mathrm{m}^{2}\right.$ (underweight); $18.5-24.9 \mathrm{~kg} / \mathrm{m}^{2}$ (normal weight, reference category); $25-29.9 \mathrm{~kg} / \mathrm{m}^{2}$ (overweight) and $\geq 30 \mathrm{~kg} / \mathrm{m}^{2}$ (obese)] as explanatory variables, with adjustment for age at diagnosis $(<35,35-45,45-55,55-65,65-75,>75$ years $)$ and ethnicity (Chinese, Malay, Native).

The association between breast cancer subtypes and all-cause mortality/recurrence was determined using Kaplan-Meier survival curves and Cox-proportional hazards regression models, which included adjustments for standard prognostic parameters including age at diagnosis, ethnicity, BMI, histologic grade, TNM stage I-IV [i.e. size $(\mathrm{T})$, nodal status $(\mathrm{N})$ and metastasis $(\mathrm{M})$ ], systemic therapy (endocrine (tamoxifen or aromatase inhibitor (AI)) and chemotherapy), radiotherapy and surgery. Follow-up started at diagnosis of breast cancer and ended at time of event (recurrence/death) or censoring (end of follow-up or, for the recurrence analysis, also death). For all-cause mortality, we censored at 10 years because this is the threshold at which most breast cancers are, by convention, considered cured in the absence of recurrence or death. We adopted a two-step approach in our survival analyses. In the first step, each of the above risk factors was modeled separately in basic models adjusted for standard prognostic factors separately for each tumor subtype. To test for heterogeneity in risk factor and survival relationships by subtype, we included an interaction term between each risk factor and tumor subtype. Violation of the proportionality assumption of the hazard model was tested by modeling each risk factor as a time-varying covariate. In the second step, it was decided, a priori, that factors that were associated with survival with $P<0.1$ in the basic model were to be mutually adjusted for in a multivariable model that included the standard prognostic factors mentioned above. Using likelihood ratio (LR) test, we compared this model with one containing only the clinicopathological factors. For sensitivity analysis, we conducted survival analysis for women stratified into two age groups $(<50 \mathrm{yrs}$ and $\geq 50 \mathrm{yrs})$. Also, we performed additional sensitivity analysis by excluding women with stage IV disease from our multivariate analyses for both all-cause mortality and recurrence. Results were very similar from these sensitivity analyses as compared to analyses including all women and we therefore presented results from all patients. All analyses were two-sided and performed using Stata statistical software version 14.0 (StataCorp, College Station, TX, USA).

\section{Results}

In total, our analysis included 3012 invasive breast cancer cases, with a total of 579 deaths in 10 years and 314 recurrence events in 5 years. The mean age at diagnosis was 52 years and mean BMI was $25 \mathrm{~kg} / \mathrm{m}^{2}$. The majority of the patients were Chinese (48\%) and had early-stage (I and II, 56\%) and HR-positive (66\%) tumors (Table 1). Of the 3012 patients, 1016 (34\%) were luminal A-like, 989 (33\%) were luminal B-like, 387 (13\%) were 
Table 1 Distribution of risk factors and clinicopathological characteristics by tumor subtype

\begin{tabular}{|c|c|c|c|c|c|c|c|c|c|c|}
\hline Characteristic & $\begin{array}{l}\text { Overall } \\
(\%)\end{array}$ & $\begin{array}{l}\text { A-like }(N=1016 / \\
34 \%)\end{array}$ & $\%$ & $\begin{array}{l}\text { B-like }(N=989 / \\
33 \%)\end{array}$ & $\%$ & $\begin{array}{l}\text { HER2-enriched ( } N= \\
387 / 13 \%)\end{array}$ & $\%$ & $\begin{array}{l}\text { Triple-neg. }(N=620 / \\
20 \%)\end{array}$ & $\%$ & $P$ value $^{a}$ \\
\hline \multicolumn{11}{|l|}{ Age, yrs } \\
\hline Mean (range) & $\begin{array}{l}51.6(19, \\
91)\end{array}$ & $52.6(24-90)$ & & $51.1(19,90)$ & & $51.6(23,91)$ & & $51.5(21,87)$ & & \\
\hline$<35$ & $180(5)$ & 47 & 4.6 & 49 & 5.0 & 26 & 6.7 & 40 & 6.5 & 0.01 \\
\hline $35-45$ & $711(21)$ & 192 & 18.9 & 219 & 22.1 & 76 & 19.7 & 144 & 23.2 & \\
\hline $45-55$ & 1204 (36) & 368 & 36.2 & 366 & 37.0 & 136 & 35.1 & 203 & 32.7 & \\
\hline $55-65$ & $806(24)$ & 241 & 23.7 & 246 & 24.9 & 105 & 27.1 & 143 & 23.1 & \\
\hline $65-75$ & $364(11)$ & 135 & 13.3 & 90 & 9.1 & 35 & 9.1 & 65 & 10.5 & \\
\hline$>75$ & $90(3)$ & 33 & 3.3 & 19 & 1.9 & 9 & 2.3 & 25 & 4.0 & \\
\hline \multicolumn{11}{|l|}{ Ethnicity } \\
\hline Chinese & $1626(48)$ & 567 & 55.8 & 435 & 44 & 180 & 46.5 & 275 & 44.4 & $<0.0001$ \\
\hline Malay & $801(24)$ & 204 & 20.1 & 263 & 26.6 & 104 & 26.9 & 155 & 25 & \\
\hline Native & $928(28)$ & 245 & 24.1 & 291 & 29.4 & 103 & 26.6 & 190 & 30.6 & \\
\hline \multicolumn{11}{|l|}{ Menarche } \\
\hline$\leq 12 \mathrm{yrs}$ & 1105 (33) & 344 & 34.1 & 326 & 33.2 & 118 & 30.8 & 190 & 30.9 & 0.20 \\
\hline 13 yrs & 1117 (34) & 318 & 31.5 & 340 & 34.7 & 148 & 38.6 & 201 & 32.7 & \\
\hline 14 yrs & $548(16)$ & 163 & 16.2 & 167 & 17 & 60 & 15.7 & 111 & 18 & \\
\hline$\geq 15 \mathrm{yrs}$ & $559(17)$ & 184 & 18.2 & 148 & 15.1 & 57 & 14.9 & 113 & 18.4 & \\
\hline \multicolumn{11}{|l|}{ Menopause } \\
\hline$\leq 50 \mathrm{yrs}$ & $428(29)$ & 146 & 32.7 & 128 & 29.7 & 38 & 23.2 & 87 & 31.6 & 0.14 \\
\hline$>50$ yrs & $1031(71)$ & 301 & 67.3 & 303 & 70.3 & 126 & 76.8 & 188 & 68.4 & \\
\hline \multicolumn{11}{|l|}{ Parity } \\
\hline Nulliparous & $745(22)$ & 242 & 23.8 & 224 & 22.6 & 77 & 19.9 & 128 & 20.6 & 0.29 \\
\hline Parous & $2601(78)$ & 774 & 76.2 & 765 & 77.4 & 310 & 80.1 & 492 & 79.4 & \\
\hline \multicolumn{11}{|l|}{ Age at FFP ${ }^{b}$, yrs } \\
\hline$<21$ & $466(18)$ & 116 & 15 & 158 & 20.7 & 54 & 17.4 & 95 & 19.3 & 0.02 \\
\hline $21-24.9$ & 1011 (39) & 284 & 36.7 & 287 & 37.5 & 129 & 41.6 & 199 & 40.5 & \\
\hline $25-30$ & $864(33)$ & 275 & 35.5 & 248 & 32.4 & 103 & 33.2 & 154 & 31.4 & \\
\hline$>30$ & $259(10)$ & 99 & 12.8 & 72 & 9.4 & 24 & 7.7 & 43 & 8.7 & \\
\hline \multicolumn{11}{|l|}{ Breastfeeding } \\
\hline No & 377 (14) & 133 & 17.2 & 103 & 13.5 & 31 & 10 & 64 & 13 & 0.01 \\
\hline Yes & $2224(86)$ & 641 & 82.9 & 662 & 86.5 & 279 & 90 & 428 & 87 & \\
\hline \multicolumn{11}{|c|}{ Breastfeeding duration } \\
\hline$<6$ months & $927(52)$ & 285 & 54.2 & 280 & 51.8 & 111 & 49.6 & 175 & 51.3 & 0.40 \\
\hline 6-10 months & $374(21)$ & 112 & 21.3 & 122 & 22.6 & 48 & 21.4 & 62 & 18.2 & \\
\hline$>10$ months & $489(27)$ & 129 & 24.5 & 138 & 25.6 & 65 & 29.0 & 104 & 30.5 & \\
\hline \multicolumn{11}{|l|}{$\mathrm{BMI}, \mathrm{kg} / \mathrm{m}^{2}$} \\
\hline$<18.5$ & $1253(39)$ & 373 & 37.8 & 336 & 35.1 & 163 & 43.7 & 240 & 40.2 & 0.03 \\
\hline $18.5-24.9$ & $565(17)$ & 160 & 16.2 & 172 & 18 & 68 & 18.2 & 114 & 19.1 & \\
\hline $25-29.9$ & $998(31)$ & 317 & 32.2 & 301 & 31.4 & 104 & 27.9 & 177 & 29.6 & \\
\hline$\geq 30$ & $433(13)$ & 136 & 13.8 & 148 & 15.5 & 38 & 10.2 & 66 & 11.1 & \\
\hline \multicolumn{11}{|l|}{ Family history } \\
\hline No & $2835(86)$ & 840 & 84 & 854 & 87.8 & 338 & 88 & 516 & 84.9 & 0.05 \\
\hline Yes & 468 (14) & 160 & 16 & 119 & 12.2 & 46 & 12 & 92 & 15.1 & \\
\hline
\end{tabular}


Table 1 Distribution of risk factors and clinicopathological characteristics by tumor subtype (Continued)

\begin{tabular}{|c|c|c|c|c|c|c|c|c|c|c|}
\hline Characteristic & $\begin{array}{l}\text { Overall } \\
(\%)\end{array}$ & $\begin{array}{l}\text { A-like }(N=1016 / \\
34 \%)\end{array}$ & $\%$ & $\begin{array}{l}\text { B-like }(N=989 / \\
33 \%)\end{array}$ & $\%$ & $\begin{array}{l}\text { HER2-enriched }(N= \\
387 / 13 \%)\end{array}$ & $\%$ & $\begin{array}{l}\text { Triple-neg. }(N=620 / \\
20 \%)\end{array}$ & $\%$ & $P$ value $^{a}$ \\
\hline \multicolumn{11}{|l|}{ Histological grade } \\
\hline Well diff. & $365(11)$ & 212 & 20.9 & 67 & 6.8 & 10 & 2.6 & 27 & 4.4 & $<0.0001$ \\
\hline Moderately diff. & $1790(55)$ & 781 & 76.9 & 442 & 44.7 & 180 & 46.5 & 238 & 38.4 & \\
\hline Poorly diff. & $1123(34)$ & - & - & 473 & 47.8 & 192 & 49.6 & 344 & 55.5 & \\
\hline \multicolumn{11}{|l|}{ Stage } \\
\hline I & $454(14)$ & 222 & 22.1 & 100 & 10.3 & 34 & 8.9 & 61 & 10 & $<0.0001$ \\
\hline$\|$ & $1353(42)$ & 444 & 44.2 & 395 & 40.6 & 136 & 35.6 & 249 & 40.9 & \\
\hline III & 1005 (31) & 241 & 24 & 330 & 33.9 & 150 & 39.3 & 203 & 33.3 & \\
\hline IV & $421(13)$ & 78 & 7.8 & 139 & 14.3 & 57 & 14.9 & 91 & 14.9 & \\
\hline \multicolumn{11}{|l|}{ Tumor size } \\
\hline$<2 \mathrm{~cm}$ & 2137 (64) & 761 & 75.6 & 620 & 63.3 & 210 & 54.7 & 353 & 57.8 & $<0.0001$ \\
\hline $2-5 \mathrm{~cm}$ & $522(16)$ & 121 & 12 & 150 & 15.3 & 83 & 21.6 & 104 & 17 & \\
\hline$>5 \mathrm{~cm}$ & $660(20)$ & 125 & 12.4 & 209 & 21.4 & 91 & 23.7 & 154 & 25.2 & \\
\hline \multicolumn{11}{|l|}{ Node status } \\
\hline 0 & $1517(46)$ & 566 & 55.7 & 368 & 37.2 & 146 & 37.7 & 272 & 43.9 & $<0.0001$ \\
\hline 1 & $922(28)$ & 249 & 24.5 & 310 & 31.3 & 95 & 24.5 & 163 & 26.3 & \\
\hline 2 & $480(15)$ & 120 & 11.8 & 156 & 15.8 & 70 & 18.1 & 94 & 15.2 & \\
\hline$\geq 3$ & 375 (11) & 72 & 7.1 & 139 & 14.1 & 68 & 17.6 & 75 & 12.1 & \\
\hline \multicolumn{11}{|l|}{ Endocrine } \\
\hline None & $1248(40)$ & 48 & 5.1 & 126 & 14.2 & 357 & 97.5 & 584 & 97.2 & $<0.0001$ \\
\hline Tamoxifen & $1456(47)$ & 687 & 72.9 & 591 & 66.7 & 7 & 1.9 & 15 & 2.5 & \\
\hline $\begin{array}{l}\text { Aromatase } \\
\text { Inhibitor }\end{array}$ & 395 (13) & 207 & 22.0 & 169 & 19.1 & 2 & 0.6 & 2 & 0.3 & \\
\hline \multicolumn{11}{|l|}{ Chemotherapy } \\
\hline No & 799 (24) & 371 & 37.3 & 177 & 18.3 & 62 & 16.4 & 95 & 15.5 & $<0.0001$ \\
\hline Yes & $2483(76)$ & 625 & 62.7 & 789 & 81.7 & 317 & 83.6 & 519 & 84.5 & \\
\hline \multicolumn{11}{|l|}{ Surgery } \\
\hline No & 389 (12) & 80 & 10.2 & 106 & 15.8 & 53 & 19.4 & 87 & 16.3 & $<0.0001$ \\
\hline Yes & $2841(88)$ & 913 & 89.8 & 833 & 84.2 & 312 & 80.6 & 519 & 83.7 & \\
\hline \multicolumn{11}{|l|}{ Radiotherapy } \\
\hline No & $800(26)$ & 261 & 27.7 & 218 & 24.6 & 79 & 23.9 & 149 & 26.4 & 0.01 \\
\hline Yes & $2235(74)$ & 683 & 72.3 & 669 & 75.4 & 252 & 76.1 & 416 & 73.6 & \\
\hline
\end{tabular}

Breast cancer subtypes were defined based on 2013 St Gallen criteria by using hormone receptor (ER and PR) and HER2 in conjunction with histologic grade. In bold are statistically significant $P$ values $(<0.05)$

${ }^{\text {a }} P$ values are for chi-square tests

${ }^{\mathrm{b}}$ FFP first full-term pregnancy

HER2-enriched, and 620 (20\%) were triple-negative, respectively.

\section{Distribution of risk factors and clinicopathological characteristics by tumor subtype}

As shown in Table 1, women with the luminal A-like subtype were slightly older than those with other subtypes. The distributions of ethnicity $(P$ value $<0.001)$, age at FFP $(P$ value $=0.019)$, breastfeeding practices $(P$ value $=0.01)$, family history $(P$ value $=0.05)$ and BMI
$(P$ value $=0.03)$ differed by subtype. No differences were observed in the distributions of age at menarche, age at menopause and parity according to subtype. The frequencies of all clinicopathological parameters differed by subtype, with low-grade, small, early-stage and node-negative tumors being more frequent for the luminal A-like subtype (Table 1).

Table 2 shows the associations between examined risk factors and molecular subtype in the multivariable polytomous regression model. Compared with women with 
Table 2 OR and 95\% Cl from a polytomous logistic regression model testing the associations between breast cancer risk factors and tumor molecular subtype

\begin{tabular}{|c|c|c|c|c|c|c|c|}
\hline \multirow[t]{3}{*}{ Risk factor } & \multicolumn{7}{|l|}{ Subtype } \\
\hline & \multirow{2}{*}{$\begin{array}{l}\text { A-like (comparison group) } \\
\mathrm{N}\end{array}$} & \multicolumn{2}{|c|}{ B-like } & \multicolumn{2}{|c|}{ HER2-enriched } & \multicolumn{2}{|c|}{ Triple-negative } \\
\hline & & $\mathrm{N}$ & $\mathrm{OR}^{\mathrm{a}}(95 \% \mathrm{Cl})$ & $\mathrm{N}$ & OR (95\% Cl) & $\mathrm{N}$ & OR (95\% Cl) \\
\hline \multicolumn{8}{|l|}{ Ethnicity } \\
\hline Chinese & 567 & 435 & 1.00 (reference) & 180 & 1.00 (reference) & 275 & 1.00 (reference) \\
\hline Malay & 204 & 263 & $1.50(1.18,1.90)$ & 104 & $1.54(1.13,2.11)$ & 155 & $1.51(1.14,1.98)$ \\
\hline Native & 245 & 291 & $1.39(1.10,1.76)$ & 103 & $1.21(0.88,1.66)$ & 190 & $1.50(1.15,1.95)$ \\
\hline$P$ value & & & 0.001 & & 0.10 & & $<0.001$ \\
\hline \multicolumn{8}{|l|}{ Menarche } \\
\hline$\leq 12 \mathrm{yrs}$ & 344 & 326 & 1.00 (reference) & 118 & 1.00 (reference) & 190 & 1.00 (reference) \\
\hline 13 yrs & 318 & 340 & $1.14(0.91,1.43)$ & 148 & $1.31(0.98,1.77)$ & 201 & $1.15(0.88,1.49)$ \\
\hline $14 \mathrm{yrs}$ & 163 & 167 & $1.04(0.79,1.38)$ & 60 & $1.02(0.70,1.48)$ & 111 & $1.18(0.86,1.61)$ \\
\hline$\geq 15$ yrs & 184 & 148 & $0.83(0.63,1.10)$ & 57 & $0.87(0.59,1.28)$ & 113 & $1.10(0.80,1.50)$ \\
\hline$P$ value & & & 0.43 & & 0.51 & & 0.31 \\
\hline \multicolumn{8}{|l|}{ Parity and $\mathrm{BF}^{\mathrm{b}}$} \\
\hline Nulliparous & 242 & 224 & 1.00 (reference) & 77 & 1.00 (reference) & 128 & 1.00 (reference) \\
\hline Parous and No BF & 133 & 103 & $1.26(0.84,1.90)$ & 31 & $1.07(0.76,1.52)$ & 64 & $1.25(0.77,2.00)$ \\
\hline Parous and BF & 641 & 662 & $1.44(1.05,1.98)$ & 279 & $1.64(1.06,2.55)$ & 428 & $1.54(1.07,2.22)$ \\
\hline$P$ value & & & 0.17 & & 0.005 & & 0.12 \\
\hline \multicolumn{8}{|l|}{ Age at FFP' } \\
\hline$<21$ yrs & 116 & 158 & 1.00 (reference) & 54 & 1.00 (reference) & 95 & 1.00 (reference) \\
\hline $21-24.9$ yrs & 284 & 287 & $0.78(0.57,1.05)$ & 129 & $1.01(0.67,1.51)$ & 199 & $0.90(0.64,1.26)$ \\
\hline $25-30$ yrs & 275 & 248 & $0.77(0.57,1.06)$ & 103 & $0.91(0.60,1.38)$ & 154 & $0.77(0.54,1.09)$ \\
\hline$>30$ yrs & 99 & 72 & $0.63(0.42,0.94)$ & 24 & $0.57(0.32,1.02)$ & 43 & $0.58(0.36,0.93)$ \\
\hline$P$ value & & & 0.35 & & 0.06 & & 0.38 \\
\hline \multicolumn{8}{|l|}{ Family history } \\
\hline No & 840 & 854 & 1.00 (reference) & 338 & 1.00 (reference) & 516 & 1.00 (reference) \\
\hline Yes & 160 & 119 & $0.72(0.55,0.94)$ & 46 & $0.71(0.49,1.02)$ & 92 & $0.92(0.69,1.23)$ \\
\hline$P$ value & & & 0.02 & & 0.05 & & 0.62 \\
\hline \multicolumn{8}{|l|}{$\mathrm{BMI}, \mathrm{kg} / \mathrm{m}^{2}$} \\
\hline $18.5-24.9$ & 160 & 172 & 1.00 (reference) & 68 & 1.00 (reference) & 114 & 1.00 (reference) \\
\hline$<18.5$ & 373 & 336 & $0.89(0.69,1.17)$ & 163 & $1.07(0.76,1.51)$ & 240 & $0.96(0.72,1.29)$ \\
\hline$P$ value & & & 0.38 & & 0.65 & & 0.76 \\
\hline $25-29.9$ & 317 & 301 & $0.86(0.65,1.12)$ & 104 & $0.74(0.51,1.06)$ & 177 & $0.75(0.55,1.02)$ \\
\hline$P$ value & & & 0.27 & & 0.13 & & 0.08 \\
\hline$\geq 30$ & 136 & 148 & $0.90(0.65,1.24)$ & 38 & $0.55(0.34,0.89)$ & 66 & $0.59(0.40,0.88)$ \\
\hline$P$ value & & & 0.62 & & 0.02 & & 0.01 \\
\hline
\end{tabular}

Statistically significant $(P$ value $<0.05)$ estimates are indicated in bold

${ }^{a} \mathrm{OR}$ and corresponding estimates are from a single polytomous logistic regression model that was mutually adjusted for ethnicity, menarche, parity and breastfeeding, age at FFP, family history, BMI and age

${ }^{\mathrm{b}} B F$ breastfeeding

${ }^{c} F F P$ first full-term pregnancy

the luminal A-like subtype, women with the luminal B-like, HER2-enriched and triple-negative tumors were significantly more likely to be Malay and Native than Chinese. Furthermore, women with other tumor subtypes were more likely to be parous and have breastfed [odds ratio $(\mathrm{OR})(95 \% \mathrm{CI})$ parity and breastfeeding vs nulliparity $=1.44(1.05,1.98) ; 1.64(1.06,2.25)$; and $1.54(1.07,2.22)$ for luminal B, HER2-enriched and 
triple-negative subtypes, respectively] and less likely to experience their FFP after the age of 30 years [OR $(95 \%$ $\mathrm{CI})>30$ years vs $<21$ years $=0.63(0.42,0.94) ; 0.57(0.32$, $1.02)$; and $0.58(0.36,0.93)$ for luminal B, HER2-enriched and triple-negative subtypes, respectively] than those with the luminal A-like subtype. Women with HER2-enriched [OR (95\% CI) BMI > $30 \mathrm{~kg} / \mathrm{m}^{2}$ vs $18.5-$ $24.9 \mathrm{~kg} / \mathrm{m}^{2}=0.55 \quad(0.34,0.89) ; \quad P \quad$ value $\left.=0.02\right]$ and triple-negative [OR $(95 \% \mathrm{CI}) \mathrm{BMI}>30 \mathrm{~kg} / \mathrm{m}^{2}$ vs $18.5-$ $24.9 \mathrm{~kg} / \mathrm{m}^{2}=0.59(0.40,0.88) ; P$ value $\left.=0.01\right]$ tumors were significantly less likely to be obese than those with the luminal A-like subtype.

\section{Breast cancer risk factors in relation to all-cause mortality and recurrence by subtype}

Overall, all-cause mortality and recurrence differed significantly by tumor subtype. In general, women with luminal A-like tumors had better survival outcomes than those with the other subtypes (Fig. 1). As shown in Table 3, in basic models for each risk factor (with adjustment for standard prognostic factors in addition to age, ethnicity and BMI), later age at menarche, parity/breastfeeding, and being underweight were significantly associated with 10-year all-cause mortality in the luminal A-like but not any of the other subtypes. Also, later age at FFP showed a suggestive association with mortality in luminal A-like patients $(P$ trend $=0.08)$ but not the other subtypes. Results were similar in basic models for recurrence (Table 4).

In the multivariable model with the mutual adjustment for ethnicity, menarche, parity/breastfeeding, age at FFP, family history, and BMI in addition to standard clinicopathological factors and treatment variables, increasing age at menarche [hazard ratio (HR) (95\% confidence interval $(\mathrm{CI}) \geq 15$ years vs $\leq 12$ years $=2.28(1.05,4.95)$;
$P$ value for trend $(P$ trend $)=0.06]$; parity $/$ breastfeeding $[\mathrm{HR}(95 \% \mathrm{CI})$ vs nulliparity $=0.48(0.27,0.85) ; P$ trend $=$ $0.01]$; older age at FFP $[\mathrm{HR}(95 \% \mathrm{CI})>30$ vs $<21$ years $=$ $0.20(0.04,0.90) ; P$ trend $=0.06]$; and being underweight $[$ HR $(95 \% \mathrm{CI})$ vs normal weight $=3.46(1.21,9.89) ; P$ value $=0.02]$ or overweight $[\mathrm{HR}(95 \% \mathrm{CI})$ vs normal weight $=$ $3.14(1.04,9.50) ; P$ value $=0.04]$ remained significantly as sociated with 10-year all-cause mortality in women with the luminal A-like subtype (Table 5 and Fig. 2). For these women, the addition of age at menarche, parity/breastfeeding, and BMI, provided significantly better fit to a model containing clinicopathological factors alone $\left[\mathrm{LRX}^{2}\right.$ $(8 d f)=21.78 ; P$ value $=0.005]$. In general, the results were consistent in relation to recurrence (Table 5).

When we examined the association between duration of breastfeeding and all-cause-mortality/recurrence for luminal A-like cases with complete information on breastfeeding duration $(N=719)$, we observed an inverse association between each breastfeeding duration category and all-cause mortality [HR $(95 \% \mathrm{CI})$ vs nulliparity $=0.37(0.18,0.85), 0.86(0.35,2.11), 0.53(0.24,1.17)$ for $<6,6-10$, and $>10$ months, respectively $(\mathrm{P}$ trend $=$ $0.38)]$ and recurrence $[\mathrm{HR}(95 \% \mathrm{CI})$ vs nulliparity $=0.47$ $(0.19,1.16), 0.73(0.23,2.37), 0.05(0.01,0.42)$ for $<6,6-$ 10 , and $>10$ months, respectively $(P$ trend $=0.002)$. Among women who breastfed, all-cause mortality did not significantly vary by breastfeeding durations (comparing $>10$ months to $<6$ months, $P$ value $=0.38$ ) but women who breastfed for $>10$ months tended to have better recurrence outcomes [HR $(95 \% \mathrm{CI})$ vs $<6$ months $=0.11(0.01,0.93) ; P$ value $=0.04]$.

\section{Discussion}

In this study involving over 3000 invasive breast cancer cases from a population-based case series in Sarawak,
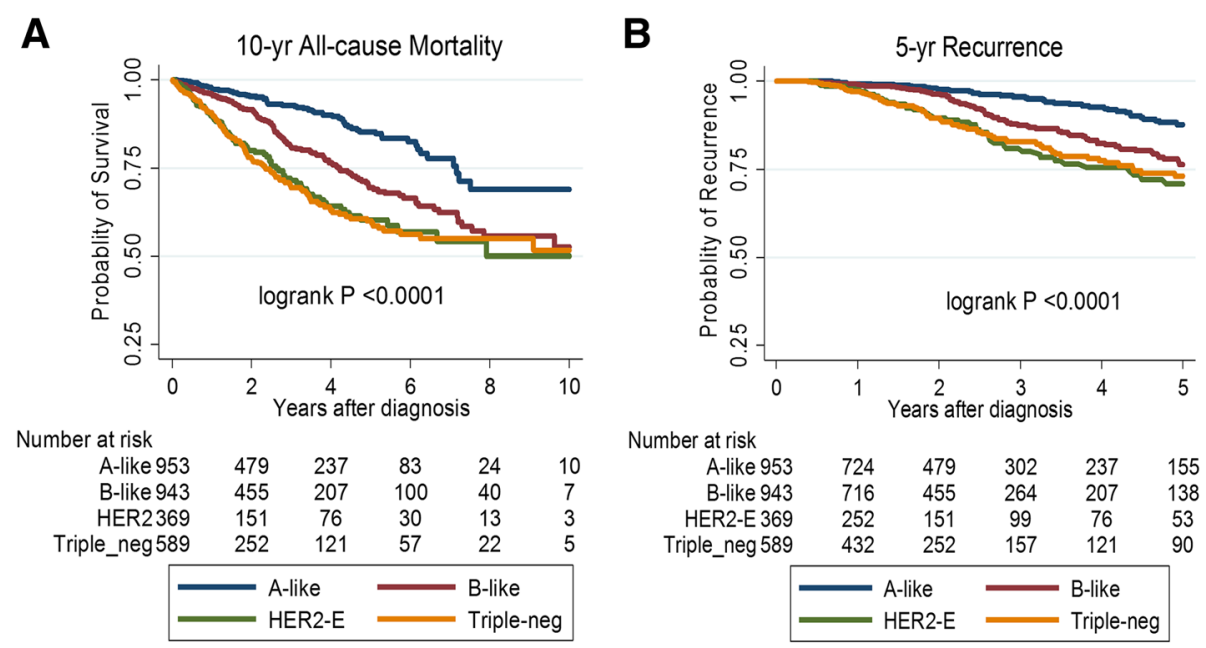

Fig. 1 Kaplan-Meier curves for the associations between breast cancer molecular subtypes and (a) 10-year all-cause mortality (b) 5-year recurrence-free survival among 3012 women diagnosed and treated in Sarawak General Hospital, Sarawak Malaysia 
Table $3 \mathrm{HR}$ and 95\% Cl for the associations between risk factors and 10-year all-cause mortality by tumor molecular subtype

\begin{tabular}{|c|c|c|c|c|c|c|c|c|c|}
\hline \multirow[t]{3}{*}{ Risk factor ${ }^{1 a}$} & \multicolumn{8}{|c|}{ 10-year all-cause mortality } & \multirow[t]{3}{*}{ P het ${ }^{\mathrm{C}}$} \\
\hline & \multicolumn{2}{|l|}{ A-like } & \multicolumn{2}{|l|}{ B-like } & \multicolumn{2}{|c|}{ HER2-enriched } & \multicolumn{2}{|c|}{ Triple-negative } & \\
\hline & N/events & HR $(95 \% \mathrm{Cl})$ & N/events & HR $(95 \% \mathrm{Cl})$ & N/events & HR (95\% Cl) & N/events & $\mathrm{HR}(95 \% \mathrm{Cl})$ & \\
\hline \multicolumn{10}{|l|}{ Ethnicity } \\
\hline Chinese & $567 / 39$ & 1.00 (reference) & $435 / 41$ & 1.00 (reference) & $180 / 38$ & 1.00 (reference) & $275 / 57$ & 1.00 (reference) & 0.81 \\
\hline Malay & $204 / 17$ & $1.23(0.65,2.34)$ & $263 / 70$ & $2.32(1.49,3.60)$ & $104 / 29$ & $1.11(0.63,1.96)$ & $155 / 54$ & $1.22(0.79,1.89)$ & \\
\hline Native & $245 / 19$ & $1.04(0.52,2.05)$ & $291 / 39$ & $1.68(1.02,2.77)$ & $103 / 20$ & $0.93(0.48,1.79)$ & $190 / 41$ & $1.06(0.67,1.67)$ & \\
\hline$P$ value & & 0.80 & & 0.03 & & 0.87 & & 0.77 & \\
\hline \multicolumn{10}{|l|}{ Menarche } \\
\hline$\leq 12 \mathrm{yrs}$ & $344 / 14$ & 1.00 (reference) & $326 / 52$ & 1.00 (reference) & $118 / 23$ & 1.00 (reference) & $190 / 46$ & 1.00 (reference) & 0.06 \\
\hline 13 yrs & $318 / 26$ & $1.53(0.76,3.11)$ & $340 / 44$ & $0.88(0.55,1.40)$ & $148 / 29$ & $0.84(0.45,1.57)$ & $201 / 46$ & $1.21(0.76,1.92)$ & \\
\hline 14 yrs & $163 / 11$ & $1.25(0.54,2.91)$ & $167 / 27$ & $0.86(0.50,1.48)$ & $60 / 22$ & $0.92(0.47,1.80)$ & $111 / 26$ & $1.05(0.61,1.80)$ & \\
\hline$\geq 15$ yrs & $184 / 23$ & $2.25(1.06,4.78)$ & $148 / 27$ & $0.84(0.49,1.42)$ & $57 / 11$ & $0.49(0.21,1.11)$ & $113 / 31$ & $0.89(0.52,1.55)$ & \\
\hline$P$ value & & 0.06 & & 0.49 & & 0.15 & & 0.66 & \\
\hline \multicolumn{10}{|l|}{ Parity and $\mathrm{BF}^{\mathrm{b}}$} \\
\hline Nulliparous & $242 / 24$ & 1.00 (reference) & $224 / 36$ & 1.00 (reference) & $77 / 18$ & 1.00 (reference) & $128 / 40$ & 1.00 (reference) & 0.28 \\
\hline Parous and No BF & $133 / 10$ & $0.59(0.27,1.32)$ & $103 / 12$ & $0.81(0.37,1.75)$ & $31 / 8$ & $0.87(0.34,2.25)$ & $64 / 20$ & $1.02(0.52,1.96)$ & \\
\hline Parous and BF & $641 / 41$ & $0.46(0.26,0.81)$ & $662 / 102$ & $0.99(0.64,1.56)$ & $279 / 61$ & $0.61(0.33,1.14)$ & $428 / 92$ & $0.86(0.55,1.34)$ & \\
\hline$P$ value & & 0.009 & & 0.91 & & 0.11 & & 0.48 & \\
\hline \multicolumn{10}{|l|}{ Age at FFPC } \\
\hline$<21$ yrs & $116 / 10$ & 1.00 (reference) & $158 / 32$ & 1.00 (reference) & $54 / 13$ & 1.00 (reference) & $95 / 19$ & 1.00 (reference) & 0.22 \\
\hline $21-24.9$ yrs & $284 / 14$ & $0.99(0.38,2.55)$ & $287 / 33$ & $1.30(0.75,2.25)$ & $129 / 28$ & $0.83(0.37,1.84)$ & $199 / 42$ & $0.71(0.39,1.29)$ & \\
\hline $25-30$ yrs & $275 / 23$ & $0.85(0.41,1.73)$ & $248 / 39$ & $1.14(0.69,1.90)$ & $103 / 22$ & $0.77(0.40,1.49)$ & $154 / 41$ & $0.98(0.60,1.60)$ & \\
\hline$>30 \mathrm{yrs}$ & $99 / 4$ & $0.36(0.10,1.29)$ & $72 / 10$ & $1.34(0.64,2.81)$ & $24 / 6$ & $0.78(0.25,2.46)$ & $43 / 9$ & $0.53(0.23,1.24)$ & \\
\hline$P$ value & & 0.08 & & 0.92 & & 0.15 & & 0.58 & \\
\hline \multicolumn{10}{|l|}{ Family history } \\
\hline No & $840 / 56$ & 1.00 (reference) & $854 / 131$ & 1.00 (reference) & $338 / 73$ & 1.00 (reference) & $516 / 122$ & 1.00 (reference) & 0.25 \\
\hline Yes & $160 / 17$ & $1.70(0.93,3.08)$ & $119 / 17$ & $1.05(0.59,1.88)$ & $46 / 13$ & $1.47(0.76,2.82)$ & $92 / 22$ & $0.77(0.46,1.27)$ & \\
\hline$P$ value & & 0.08 & & 0.86 & & & & & \\
\hline \multicolumn{10}{|l|}{$\mathrm{BMI}, \mathrm{kg} / \mathrm{m}^{2}$} \\
\hline $18.5-24.9$ & $160 / 6$ & 1.00 (reference) & $172 / 25$ & 1.00 (reference) & $68 / 15$ & 1.00 (reference) & $114 / 25$ & 1.00 (reference) & 0.80 \\
\hline$<18.5$ & $373 / 38$ & $3.42(1.20,9.71)$ & $336 / 43$ & $1.06(0.62,1.82)$ & $163 / 37$ & $0.83(0.44,1.57)$ & $240 / 63$ & $1.15(0.70,1.91)$ & \\
\hline$P$ value & & 0.02 & & 0.83 & & 0.58 & & 0.56 & \\
\hline $25-30$ & $317 / 21$ & $2.88(0.97,8.59)$ & $301 / 49$ & $1.30(0.77,2.20)$ & $104 / 19$ & $0.80(0.39,1.64)$ & $177 / 40$ & $0.92(0.54,1.57)$ & \\
\hline$P$ value & & 0.06 & & 0.32 & & 0.54 & & 0.77 & \\
\hline$>30$ & $136 / 7$ & $1.30(0.37,4.52)$ & $148 / 23$ & $1.12(0.61,2.05)$ & $38 / 10$ & $1.23(0.52,2.90)$ & $66 / 18$ & $1.21(0.63,2.32)$ & \\
\hline$P$ value & & 0.68 & & 0.71 & & 0.63 & & 0.57 & \\
\hline
\end{tabular}

In bold are variables which met our criteria $(P$ value $<0.1)$ for inclusion in multivariate models

aEach risk factor was adjusted for age, ethnicity, BMI, tumor stage, histologic grade, surgery, systemic therapy (endocrine (tamoxifen or Al versus none),

chemotherapy (any regimen versus none)) and radiotherapy (received versus none)

${ }^{\mathrm{b}} B F$ breastfeeding

${ }^{c} F F P$ first full-term pregnancy

${ }^{d} P$ value for heterogeneity (P-het) of HR estimates according to molecular subtypes

Malaysia, with detailed demographic, risk factor, pathology, and follow-up data, we investigated several established breast cancer risk factors in relation to tumor subtypes and patient outcomes. We found differences in the prevalence of parity and breastfeeding, age at FFP, family history of breast cancer and obesity across different breast tumor subtypes. In general, traditional breast cancer risk factors (older age at FFP, higher BMI, lower 
Table $4 \mathrm{HR}$ and 95\% Cl for the associations between risk factors and 5-year recurrence by tumor molecular subtype

\begin{tabular}{|c|c|c|c|c|c|c|c|c|c|}
\hline \multirow[t]{3}{*}{ Risk factor ${ }^{a}$} & \multicolumn{8}{|c|}{ Recurrence after 5 years } & \multirow{3}{*}{$\begin{array}{l}\text { P- } \\
\text { het }^{d}\end{array}$} \\
\hline & \multicolumn{2}{|l|}{ A-like } & \multicolumn{2}{|l|}{ B-like } & \multicolumn{2}{|c|}{ HER2-enriched } & \multicolumn{2}{|c|}{ Triple-negative } & \\
\hline & N/events & HR $(95 \% \mathrm{Cl})$ & N/events & HR $(95 \% \mathrm{Cl})$ & N/events & HR $(95 \% \mathrm{Cl})$ & N/events & HR $(95 \%$ Cl) & \\
\hline \multicolumn{10}{|l|}{ Ethnicity } \\
\hline Chinese & $567 / 34$ & 1.00 (reference) & $435 / 49$ & 1.00 (reference) & $180 / 30$ & 1.00 (reference) & $275 / 38$ & 1.00 (reference) & 0.96 \\
\hline Malay & $204 / 8$ & $1.01(0.39,2.60)$ & $263 / 30$ & $0.81(0.45,1.45)$ & $104 / 17$ & $0.69(0.31,1.51)$ & $155 / 24$ & $1.11(0.61,2.02)$ & \\
\hline Native & $245 / 16$ & $1.17(0.49,2.79)$ & $291 / 22$ & $0.84(0.46,1.55)$ & $103 / 10$ & $0.47(0.18,1.23)$ & $190 / 22$ & $0.67(0.34,1.34)$ & \\
\hline$P$ value & & 0.75 & & 0.55 & & 0.11 & & 0.32 & \\
\hline \multicolumn{10}{|l|}{ Menarche } \\
\hline$\leq 12 \mathrm{yrs}$ & $344 / 13$ & 1.00 (reference) & $326 / 38$ & 1.00 (reference) & $118 / 14$ & 1.00 (reference) & 190/34 & 1.00 (reference) & 0.07 \\
\hline 13 yrs & $318 / 17$ & $1.55(0.57,4.23)$ & $340 / 31$ & $1.03(0.58,1.82)$ & $148 / 21$ & $1.52(0.69,3.33)$ & $201 / 24$ & $0.72(0.38,1.37)$ & \\
\hline $14 \mathrm{yrs}$ & $163 / 14$ & $2.51(0.86,7.33)$ & $167 / 21$ & $0.81(0.40,1.64)$ & $60 / 9$ & $0.56(0.16,1.92)$ & $111 / 13$ & $0.65(0.30,1.43)$ & \\
\hline$\geq 15 \mathrm{yrs}$ & $184 / 13$ & $3.26(1.08,9.92)$ & $148 / 11$ & $0.77(0.35,1.68)$ & $57 / 12$ & $1.11(0.38,3.28)$ & $113 / 12$ & $0.61(0.27,1.36)$ & \\
\hline$P$ value & & 0.02 & & 0.41 & & 0.81 & & 0.18 & \\
\hline \multicolumn{10}{|l|}{ Parity and $\mathrm{BF}^{\mathrm{C}}$} \\
\hline Nulliparous & $242 / 19$ & 1.00 (reference) & $224 / 16$ & 1.00 (reference) & $77 / 12$ & 1.00 (reference) & $128 / 19$ & 1.00 (reference) & 0.56 \\
\hline Parous and No BF & $133 / 7$ & $0.55(0.20,1.50)$ & $103 / 14$ & $1.86(0.76,4.56)$ & $31 / 7$ & $1.35(0.39,4.64)$ & $64 / 17$ & $1.57(0.67,3.66)$ & \\
\hline Parous and BF & $641 / 32$ & $0.27(0.12,0.58)$ & $662 / 71$ & $1.63(0.81,3.28)$ & $279 / 38$ & $0.58(0.25,1.34)$ & $428 / 48$ & $0.77(0.39,1.52)$ & \\
\hline$P$ value & & 0.001 & & 0.23 & & 0.15 & & 0.28 & \\
\hline \multicolumn{10}{|l|}{ Age at FFP } \\
\hline$<25$ & $400 / 13$ & 1.00 (reference) & $445 / 44$ & 1.00 (reference) & $183 / 30$ & 1.00 (reference) & $294 / 41$ & 1.00 (reference) & 0.52 \\
\hline$\geq 25$ & $374 / 26$ & $1.69(0.66,4.34)$ & $320 / 41$ & $1.32(0.78,2.22)$ & $127 / 15$ & $0.50(0.21,1.16)$ & $197 / 23$ & $0.75(0.41,1.39)$ & \\
\hline$P$ value & & 0.27 & & 0.28 & & 0.10 & & 0.36 & \\
\hline \multicolumn{10}{|l|}{ Family history } \\
\hline No & $840 / 42$ & 1.00 (reference) & $854 / 85$ & 1.00 (reference) & $338 / 49$ & 1.00 (reference) & $516 / 66$ & 1.00 (reference) & 0.94 \\
\hline Yes & $160 / 13$ & $0.77(0.29,2.03)$ & $119 / 14$ & $1.17(0.60,2.28)$ & $46 / 7$ & $1.24(0.45,3.35)$ & $92 / 12$ & $0.85(0.43,1.70)$ & \\
\hline$P$ value & & 0.60 & & 0.64 & & 0.67 & & & \\
\hline \multicolumn{10}{|l|}{$\mathrm{BMI}, \mathrm{kg} / \mathrm{m}^{2}$} \\
\hline $18.5-24.9$ & $160 / 6$ & 1.00 (referent) & $172 / 14$ & 1.00 (referent) & $68 / 11$ & 1.00 (referent) & $114 / 16$ & 1.00 (referent) & 0.98 \\
\hline$<18.5$ & $373 / 30$ & $3.97(0.91,17.34)$ & $336 / 29$ & $1.37(0.62,3.03)$ & $163 / 21$ & $0.50(0.20,1.27)$ & $240 / 30$ & $1.01(0.51,2.01)$ & \\
\hline$P$ value & & 0.06 & & 0.43 & & 0.15 & & 0.97 & \\
\hline $25-30$ & $317 / 16$ & $3.40(0.75,15.42)$ & $301 / 37$ & $2.02(0.95,4.29)$ & $104 / 15$ & $0.70(0.27,1.81)$ & $177 / 24$ & $0.83(0.39,1.73)$ & \\
\hline$P$ value & & 0.11 & & 0.06 & & 0.46 & & 0.62 & \\
\hline$>30$ & $136 / 5$ & $1.65(0.27,10.15)$ & $148 / 15$ & $1.28(0.53,3.12)$ & $38 / 7$ & $1.41(0.46,4.27)$ & $66 / 13$ & $0.89(0.35,2.25)$ & \\
\hline$P$ value & & 0.59 & & 0.58 & & 0.55 & & 0.81 & \\
\hline
\end{tabular}

In bold are variables which met our criteria $(P$-value $<0.1)$ for inclusion in multivariate models

aEach risk factor was adjusted for age, ethnicity, BMl, tumor stage, histologic grade, surgery, systemic therapy (endocrine (tamoxifen or Al versus none),

chemotherapy (any regimen versus none)) and radiotherapy (received versus none)

${ }^{\mathrm{b}} B F$ breastfeeding

${ }^{C}$ FFP first full-term pregnancy. Due to sample size considerations age at FFP was dichotomized

${ }^{\mathrm{d}} P$ value for heterogeneity (P-het) of HR estimates according to molecular subtypes

parity, lack of breastfeeding) seem to show higher frequencies among women with the luminal A-like subtype compared with women with other subtypes. Further, we found that age at menarche, breastfeeding, and BMI were independent prognostic factors for both overall mortality and breast cancer recurrence but only for women with the luminal A-like subtype, which had better survival and recurrence outcomes than the other subtypes.

Our findings that nulliparity and older age at FFP were more prevalent in luminal A-like patients are consistent with those reported in studies in Western countries $[16,27]$. However, unlike the observation of higher BMI and shorter breastfeeding duration in triple-negative 

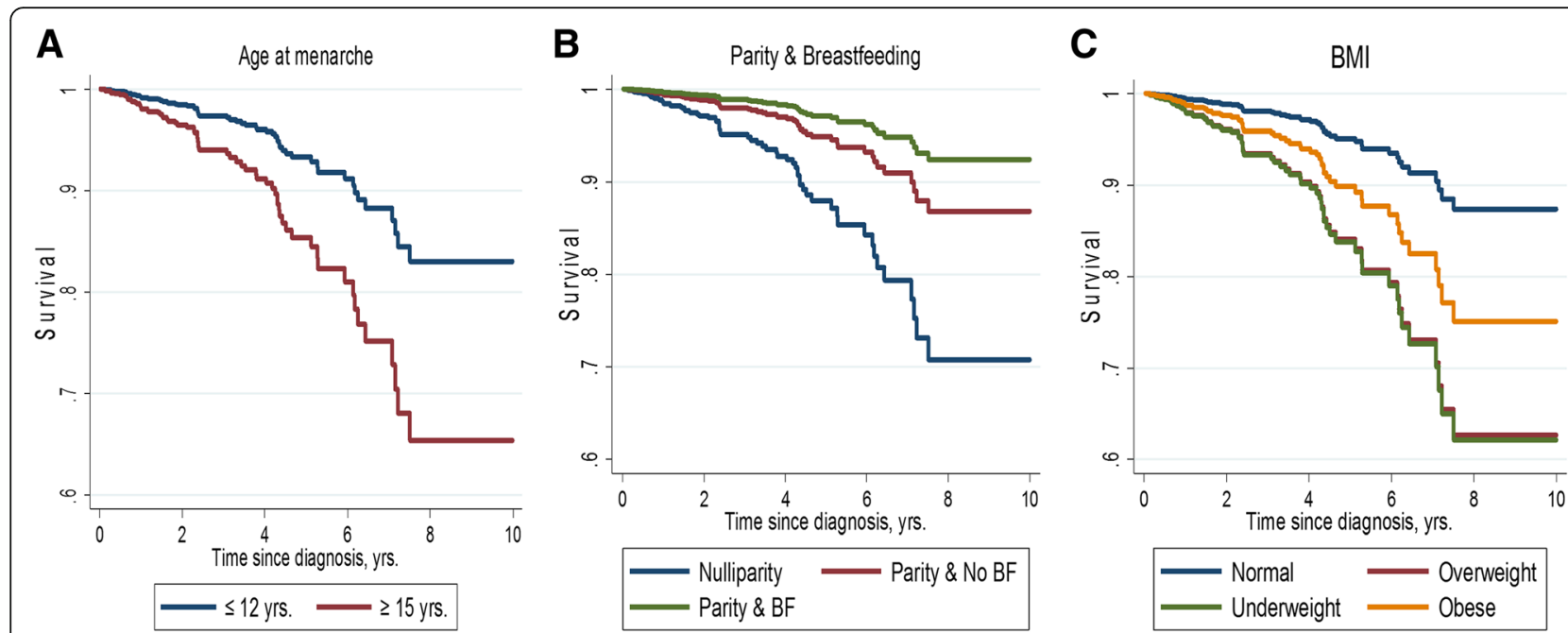

Fig. 2 Survival curves for the multivariate association between (a) age at menarche, (b) parity and breastfeeding and (c) BMI and 10-year all-cause mortality among 1016 women with the luminal A-like subtype of breast cancer

patients among Western, especially African American, women $[18,19,28,29]$, we found lower frequencies of obesity and breastfeeding among $\mathrm{HR}^{-}$(HER2-enriched and triple-negative) than luminal A-like tumors, which may be reflective of population/ethnic differences. In line with this hypothesis, a previous study conducted in South Korea [30] also showed a higher frequency of breastfeeding among women with luminal $\mathrm{B}$ or HER2-enriched than those with luminal A disease. In another study involving 730 Mexican women with breast cancer, Martinez and colleagues [31] reported the prevalence of breastfeeding to be higher among women with triple-negative than luminal A tumors. Similarly, results from a multiethnic study showed an inverse association between triple-negative tumors and breastfeeding in White, Hispanic and African American but, notably, not in Asian women, for whom breastfeeding for $>2$ months was associated with an $86 \%$ increased likelihood of triple-negative tumors [32].

The prevalence of obesity is still much lower in most Asian populations compared with other race/ethnicity groups. In contrast to the reduced breast cancer risk associated with higher BMI among premenopausal Western women, obesity is associated with increased risk for both premenopausal and postmenopausal Asian women [33-35]. The heterogeneity of obesity by tumor subtype among Asian cases remains unclear. Results from both our study and the South Korean study [30] suggest that obesity was less frequent among patients with HER2-enriched tumors. In combination with our finding that women with the HER2-enriched subtype were more likely to be parous and to have breastfed than women with the luminal A-like subtype, our data suggest that these factors (parity, breastfeeding, and low BMI) may not protect against HER2-enriched breast cancers. The decreasing prevalence of these factors associated with the adoption of westernized lifestyles may, therefore, not affect the incidence of this subtype, which is known to be more prevalent among Asian women [36, 37]. More research to understand the risk factors associated with the HER2-enriched subtype is warranted.

Most epidemiological studies considering the prognostic significance of age at menarche in breast cancer have treated the disease as a homogeneous entity, and results from these studies are largely conflicting $[1-3,8,38]$. We found that older age at menarche was associated with worse prognosis but only among women with the luminal A-like subtype. This finding is consistent with results from a previous study of women in East Asia [20] that also evaluated relationships between risk factors and survival according to subtypes. It is unclear why late menarche and younger age at FFP lead to worse survival outcomes in women with the luminal A-like subtype since they are well known protective factors in terms of breast cancer risk. One possibility is that because early menarche and late age at FFP increase breast cancer risk through prolonged and sustained exposure of the mammary epithelium to the mitogenic effects of reproductive hormones $[39,40]$, these factors predispose more strongly to $\mathrm{HR}^{+}$tumors which have better prognosis than $\mathrm{HR}^{-}$tumors $[18,41]$. Although this association was confined to the luminal A-like subtype, which, by definition, is $\mathrm{HR}^{+}$, expression of hormone receptors in tumors occurs in a spectrum. Whereas some tumors have very high expression levels, others have lower levels despite crossing the threshold for consideration as $\mathrm{HR}^{+}$. Due to differences in cumulative lifetime exposure to endogenous estrogens, luminal A-like tumors occurring 
Table 5 Multivariate HR and 95\% Cl for the association between breast cancer risk factors and 10-year all-cause mortality and 5-year recurrence among women with luminal A-like subtype breast cancer

\begin{tabular}{|c|c|c|c|c|}
\hline \multirow[t]{2}{*}{ Risk factor } & \multicolumn{2}{|c|}{ 10-year all-cause mortality } & \multicolumn{2}{|c|}{ 5-year recurrence } \\
\hline & N/events & $\mathrm{HR}^{1}(95 \% \mathrm{Cl})$ & N/events & $\mathrm{HR}^{\mathrm{a}}(95 \% \mathrm{Cl})$ \\
\hline \multicolumn{5}{|l|}{ Ethnicity } \\
\hline Chinese & $539 / 38$ & 1.00 (reference) & $399 / 21$ & 1.00 (reference) \\
\hline Malay & 194/16 & $1.14(0.59,2.20)$ & $154 / 7$ & $0.81(0.29,2.27)$ \\
\hline Native & $235 / 17$ & $0.87(0.41,1.85)$ & 190/11 & $0.90(0.35,2.29)$ \\
\hline$P$ value & & 0.95 & & 0.74 \\
\hline \multicolumn{5}{|l|}{ Menarche } \\
\hline$\leq 12$ yrs & $338 / 13$ & 1.00 (reference) & $260 / 8$ & 1.00 (reference) \\
\hline 13 yrs & $302 / 25$ & $1.45(0.70,3.01)$ & $235 / 13$ & $1.69(0.59,4.81)$ \\
\hline 14 yrs & $156 / 10$ & $1.23(0.52,2.92)$ & $119 / 8$ & $2.64(0.87,7.99)$ \\
\hline$\geq 15 \mathrm{yrs}$ & $172 / 23$ & $2.28(1.05,4.95)$ & $129 / 10$ & $3.52(1.10,11.23)$ \\
\hline$P$ value & & 0.06 & & 0.02 \\
\hline \multicolumn{5}{|l|}{ Parity and $\mathrm{BF}^{\mathrm{b}}$} \\
\hline Nulliparous & $229 / 23$ & 1.00 (reference) & $180 / 15$ & 1.00 (reference) \\
\hline Parous and No BF & $127 / 9$ & $0.61(0.26,1.42)$ & $87 / 5$ & 0.53 (0.17. 1.59) \\
\hline Parous and BF & $612 / 39$ & $0.48(0.27,0.85)$ & $476 / 19$ & $0.28(0.13,0.64)$ \\
\hline$P$ value & & 0.01 & & 0.002 \\
\hline \multicolumn{5}{|l|}{ Age at FFP' } \\
\hline$<21$ yrs & $111 / 9$ & 1.00 (reference) & $85 / 4$ & 1.00 (reference) \\
\hline $21-24.9$ yrs & $276 / 14$ & $0.85(0.31,2.31)$ & $220 / 4$ & $0.40(0.06,2.28)$ \\
\hline $25-30$ yrs & $262 / 22$ & $0.71(0.27,1.82)$ & $201 / 17$ & $1.26(0.30,5.22)$ \\
\hline$>30 \mathrm{yrs}$ & $95 / 4$ & $0.20(0.04,0.90)$ & $73 / 1$ & $0.26(0.02,3.09)$ \\
\hline$P$ value & & 0.06 & & 0.95 \\
\hline \multicolumn{5}{|l|}{ Family history } \\
\hline No & $804 / 53$ & 1.00 (reference) & $629 / 31$ & 1.00 (referent) \\
\hline Yes & $155 / 17$ & $1.68(0.91,3.10)$ & $122 / 7$ & $0.75(0.28,2.02)$ \\
\hline$P$ value & & 0.10 & & 0.57 \\
\hline \multicolumn{5}{|l|}{ BMI } \\
\hline $18.5-24.9 \mathrm{~kg} / \mathrm{m}^{2}$ & $160 / 6$ & 1.00 (reference) & $117 / 2$ & 1.00 (reference) \\
\hline$<18.5 \mathrm{~kg} / \mathrm{m}^{2}$ & $365 / 37$ & $3.46(1.21,9.89)$ & $285 / 22$ & $4.31(0.94,19.83)$ \\
\hline$P$ value & & 0.02 & & 0.06 \\
\hline $25-29.9 \mathrm{~kg} / \mathrm{m}^{2}$ & $310 / 21$ & $3.14(1.04,9.50)$ & $235 / 12$ & $4.74(1.00,22.64)$ \\
\hline$P$ value & & 0.04 & & 0.05 \\
\hline$\geq 30 \mathrm{~kg} / \mathrm{m}^{2}$ & $133 / 7$ & $1.38(0.39,4.86)$ & $106 / 3$ & $2.16(0.34,13.78)$ \\
\hline$P$ value & & 0.61 & & 0.41 \\
\hline
\end{tabular}

In bold are statistically significant estimates $(P$ value $<0.05)$

a $\mathrm{HR}$ was mutually adjusted for ethnicity, menarche, parity and breastfeeding, age at FFP, family history, BMI, age, TNM stage, histologic grade, surgery, systemic therapy (endocrine (tamoxifen or Al versus none), chemotherapy (any regimen vs and radiotherapy (received versus none)

${ }^{\mathrm{b}} B F$ breastfeeding

${ }^{C}$ FFP first full-term pregnancy

among women with late menarche may have lower levels of hormone receptor expression, hence worse survival/ recurrence outcomes, than those occurring among women with early menarche. Indeed, this is consistent with the finding by Song et al. that longer duration of endogenous estrogen exposure was associated with better survival [30]. On the other hand, late menarche and early age at FFP may be indicative of lower socioeconomic status (SES) which, in turn, may be reflective of less exposure to "westernized" environments/lifestyles. 
Nonetheless, adjustment of known surrogates for SES did not change the associations between these factors and survival. That late age at menarche and early age at FFP were not associated with survival/recurrence in the other subtypes may be due to the masking effect of other more aggressive tumor features, which are inherent in these subtypes.

Our findings of breastfeeding being associated with better outcomes are generally consistent with previous reports [2, 12, 42]. A distinctive feature of luminal A-like tumors defined in our analysis is the low levels of proliferation, indicated by low histologic grade. Findings from one previous study showed that the protective effect of breastfeeding on breast cancer mortality was stronger for tumors with low expression of proliferation genes [21]. In our study, breastfeeding was associated with a preponderance of lobular carcinoma and small size tumors, both of which are highly correlated with low levels of proliferation [43-45].

Results from several studies, summarized in two comprehensive reviews and meta-analyses [46, 47], are supportive of the prognostic value of BMI in breast cancer. The association between BMI and survival after breast cancer is thought to be U-shaped [47-49], with underweight and overweight/obese women more likely to suffer worse survival outcomes than their normal weight counterparts. In our study, being underweight and overweight, but not obese, were suggestively associated with worse prognosis, but this might be due to the low frequency of obesity in this population (13\%). Compared with overweight/obesity, the effect of underweight in breast cancer survival is less well-studied. Overall, our finding of an association between underweight and worse survival outcomes in breast cancer is in line with those of several other studies involving Asian populations [49-52]. Whilst insulin resistance, chronic inflammation, and altered adipokine and cytokine production have been proposed to underlie the obesity-cancer link [53], the precise mechanisms underpinning the relationship between underweight BMI and disease progression are not well understood. Chronic, pre-diagnostic, malnutrition may contribute to weight loss in cancer patients and may independently influence outcomes in the disease. However, when we examined the impact of indicators of socioeconomic deprivation, as surrogates for chronic malnutrition, our estimates remained unchanged.

Strengths of this study include: a population-based breast cancer case series in an understudied Asian population and the collection of detailed questionnaire information, which allowed us to account for various confounding variables including sociodemographic factors. Several limitations of this study should be noted. First, we did not include controls in our study and therefore our case-case comparisons could not be translated into relative risk estimates. Second, relatively small sample sizes for some of the subtypes may have affected our power to detect significant associations. Third, data on the specific cause of death were not available and, therefore, we only evaluated risk factors in relation to all-cause, but not breast cancer-specific, mortality even though some of the risk factors, such as BMI, are important predictors of death from other causes [54]. Nonetheless, the consistency of our results for all-cause mortality and for breast cancer recurrence indicate that these factors may contribute to breast cancer-specific mortality in a similar manner.

\section{Conclusions}

In conclusion, our data indicate that risk factors for breast cancer are differentially associated with tumor subtypes and exert subtype-specific influence on survival/recurrence from the disease. Specifically, we observed that menarche after the age of 15 years, FFP after 30 years, underweight or overweight BMI, and breastfeeding practices were associated with survival/recurrence only among women with luminal A-like tumors. These findings are supportive of the prognostic value of reproductive and lifestyle-related factors in tumors with biologically favorable profiles, and could have implications for clinical counseling and for the development of subtype-specific prognostic tools. Future prospective studies are needed to delineate the role of lifestyle modification, especially changes in BMI, in improving clinical outcomes for women with luminal A-like breast cancer.

\section{Abbreviations}

ACM: All-cause mortality; BMI: Body mass index; Cl: Confidence interval:

ER: Estrogen receptor; FFP: First full-term pregnancy; HER2: Human epidermal growth factor receptor 2; HR: Hazard ratio; IHC: Immunohistochemical; KM: Kaplan-Meier; LR: Likelihood ratio; OR: Odds ratio; PR: Progesterone receptor

\section{Acknowledgements}

The authors acknowledge Julie Buckland and Jane Demuth at Information Management Systems for data management support.

\section{Funding}

This research was supported by the Intramural Research Program of the National Institutes of Health, National Cancer Institute, Division of Cancer Epidemiology and Genetics.

Availability of data and materials

The datasets used and analyzed for the current study will be available from the corresponding author upon reasonable request.

Authors' contributions

MA, HS, and XRY conceived the study design and analytical concept. MA performed statistical analyses and drafted the manuscript. HS, RMP, and XRY contributed to the interpretation of results and the critical revision of the manuscript. DBCR and TST contributed to the acquisition of patient information and pathology data. JG helped with data management. All authors participated in the revision of the manuscript and approved the final manuscript. 


\section{Ethics approval and consent to participate}

All study participants provided written informed consent and the project was approved by the Ethics Committee of the National Institutes of Health, Malaysia. The study was also exempted from review by the Office of Human Subject Research Protections at the National Institutes of Health since NIH investigators do not have the access to the personal identifying information.

\section{Consent for publication}

Not applicable.

\section{Competing interests}

The authors declare that they have no competing interests.

\section{Publisher's Note}

Springer Nature remains neutral with regard to jurisdictional claims in published maps and institutional affiliations.

\section{Author details \\ ${ }^{1}$ Integrative Tumor Epidemiology Branch, Division of Cancer Epidemiology and Genetics, National Cancer Institute (NCl), National Institutes of Health, 9609 Medical Center Drive, Rockville, MD 20850, USA. ${ }^{2}$ Surveillance and Health Services Research, American Cancer Society, 250 Williams Street NW, Atlanta, GA 30303, USA. ${ }^{3}$ Department of Radiotherapy, Oncology and Palliative Care, Sarawak General Hospital, Kuching, Sarawak, Malaysia. ${ }^{4}$ Division of Cancer Control \& Population Sciences, National Cancer Institute, National Institutes of Health, Rockville, MD, USA.}

Received: 6 April 2018 Accepted: 31 July 2018

Published online: 18 September 2018

\section{References}

1. Caleffi M, Fentiman IS, Birkhead BG. Factors at presentation influencing the prognosis in breast cancer. Eur J Cancer Clin Oncol. 1989;25(1):51-6.

2. Trivers KF, Gammon MD, Abrahamson PE, Lund MJ, Flagg EW, Kaufman JS, et al. Association between reproductive factors and breast cancer survival in younger women. Breast Cancer Res Treat. 2007;103(1):93-102.

3. Orgéas CC, Hall P, Rosenberg LU, Czene K. The influence of menstrual risk factors on tumor characteristics and survival in postmenopausal breast cancer. Breast Cancer Res. 2008;10(6):R107.

4. Schouten LJ, Hupperets PS, Jager JJ, Volovics L, Wils JA, Verbeek AL, et al. Prognostic significance of etiological risk factors in early breast cancer. Breast Cancer Res Treat. 1997;43(3):217-23.

5. Kroman N, Wohlfahrt J, West Andersen K, Mouridsen HT, Westergaard T, Melbye M. Parity, age at first childbirth and the prognosis of primary breast cancer. Br J Cancer. 1998;78(11):1529-33.

6. Papatestas AE, Mulvihill M, Josi C, loannovich J, Lesnick G, Aufses AH. Parity and prognosis in breast cancer. Cancer. 1980;45(1):191-4.

7. Green A, Beral V, Moser K. Mortality in women in relation to their childbearing history. Br Med J. 1988;297(6645):391-5.

8. Korzeniowski S, Dyba T. Reproductive history and prognosis in patients with operable breast cancer. Cancer. 1994;74(5):1591-4.

9. Black MM, Hankey BF, Barclay THC. Parity as a prognostic factor in young breast Cancer Patients2. J Natl Cancer Inst. 1983;70(1):27-30.

10. Mohle-Boetani JC, Grosser S, Whittemore AS, Malec M, Kampert JB, Paffenbarger RS. Body size, reproductive factors, and breast cancer survival. Prev Med. 1988;17(5):634-42.

11. Olson SH, Zauber AG, Tang J, Harlap S. Relation of time since last birth and parity to survival of young women with breast Cancer. Epidemiology. 1998; 9(6):669-71.

12. Phillips K-A, Milne RL, Friedlander ML, Jenkins MA, McCredie MRE, Giles GG, et al. Prognosis of premenopausal breast Cancer and childbirth prior to diagnosis. J Clin Oncol. 2004;22(4):699-705.

13. Parker JS, Mullins M, Cheang MC, Leung S, Voduc D, Vickery T, et al. Supervised risk predictor of breast cancer based on intrinsic subtypes. J Clin Oncol. 2009;27(8):1160-7.

14. Goldhirsch A, Winer EP, Coates AS, Gelber RD, Piccart-Gebhart M, Thürlimann B, et al. Personalizing the treatment of women with early breast cancer: highlights of the St Gallen International Expert Consensus on the Primary Therapy of Early Breast Cancer 2013. Ann Oncol. 2013;24(9):2206-23.

15. Senkus E, Kyriakides S, Penault-Llorca F, Poortmans P, Thompson A, Zackrisson S, et al. Primary breast cancer: ESMO clinical practice guidelines for diagnosis, treatment and follow-up. Ann Oncol. 2013; 24(6):vi7-vi23.

16. Millikan RC, Newman B, Tse C-K, Moorman PG, Conway K, Smith LV, et al. Epidemiology of basal-like breast cancer. Breast Cancer Res Treat. 2008; 109(1):123-39.

17. Jatoi I, Anderson WF. Qualitative age interactions in breast cancer studies: a mini-review. Future Oncol. 2010;6(11):1781-8.

18. Yang XR, Chang-Claude J, Goode EL, Couch FJ, Nevanlinna H, Milne RL, et al. Associations of breast cancer risk factors with tumor subtypes: a pooled analysis from the breast Cancer association consortium studies. J Natl Cancer Inst. 2011;103(3):250-63.

19. Anderson WF, Pfeiffer RM, Wohlfahrt J, Ejlertsen B, Jensen M-B, Kroman $\mathrm{N}$. Associations of parity-related reproductive histories with $E R \pm$ and HER2 \pm receptor-specific breast cancer aetiology. Int J Epidemiol. 2017;46(1):373

20. Song N, Choi J-Y, Sung H, Jeon S, Chung S, Song M, et al. Tumor subtypespecific associations of hormone-related reproductive factors on breast Cancer survival. PLoS One. 2015;10(4):e0123994.

21. Kwan ML, Bernard PS, Kroenke CH, Factor RE, Habel LA, Weltzien EK, et al. Breastfeeding, PAM50 Tumor Subtype, and Breast Cancer Prognosis and Survival. J Natl Cancer Inst. 2015;107(7):djv087-djv.

22. Cespedes Feliciano EM, Kwan ML, Kushi LH, Chen WY, Weltzien EK, Castillo AL, et al. Body mass index, PAM50 subtype, recurrence, and survival among patients with nonmetastatic breast cancer. Cancer. 2017;123(13):2535-42.

23. Yang XR, Devi BCR, Sung $H$, Guida J, Mucaki EJ, Xiao $Y$, et al. Prevalence and spectrum of germline rare variants in BRCA1/2 and PALB2 among breast cancer cases in Sarawak, Malaysia. Breast Cancer Res Treat. 2017;165(3):687-97.

24. Devi CRB, Tang TS, Corbex M. Incidence and risk factors for breast cancer subtypes in three distinct south-east Asian ethnic groups: Chinese, Malay and natives of Sarawak, Malaysia. Int J Cancer. 2012:131(12):2869-77.

25. Tamimi RM, Colditz GA, Hazra A, Baer HJ, Hankinson SE, Rosner B, et al. Traditional breast cancer risk factors in relation to molecular subtypes of breast cancer. Breast Cancer Res Treat. 2012;131(1):159-67.

26. Mustapha A, Jenny C-C, Raza AH, Nilanjan C, Penny C, Frances D, et al. Etiology of hormone receptor positive breast cancer differs by levels of histologic grade and proliferation. Int J Cancer. 2018;143(4):746-57.

27. Anderson KN, Schwab RB, Martinez ME. Reproductive risk factors and breast cancer subtypes: a review of the literature. Breast Cancer Res Treat. 2014; 144(1):1-10.

28. Chen L, Li Cl, Tang M-TC, Porter P, Hill DA, Wiggins CL, et al. Reproductive factors and risk of luminal, HER2-overexpressing, and triple-negative breast Cancer among multiethnic women. Cancer epidemiology biomarkers \&amp. Prevention. 2016;25(9):1297-304.

29. Kwan ML, Kushi LH, Weltzien E, Maring B, Kutner SE, Fulton RS, et al. Epidemiology of breast cancer subtypes in two prospective cohort studies of breast cancer survivors. Breast Cancer Res. 2009;11(3):1-13.

30. Song N, Choi J-Y, Sung H, Chung S, Song M, Park SK, et al. Heterogeneity of epidemiological factors by breast tumor subtypes in Korean women: a case-case study. Int J Cancer. 2014;135(3):669-81.

31. Martinez ME, Wertheim BC, Natarajan L, Schwab R, Bondy ML, DaneriNavarro A, et al. Reproductive factors, heterogeneity, and breast tumor subtypes in women of Mexican descent. Cancer Epidemiol Biomarkers Prev. 2013:22(10):1853-61.

32. Shinde SS, Forman MR, Kuerer HM, Yan K, Peintinger F, Hunt KK, et al. Higher parity and shorter breastfeeding duration. Cancer. 2010;116(21): 4933-43.

33. Wada K, Nagata C, Tamakoshi A, Matsuo K, Oze I, Wakai K, et al. Body mass index and breast cancer risk in Japan: a pooled analysis of eight populationbased cohort studies. Ann Oncol. 2014;25(2):519-24.

34. Bandera EV, Maskarinec G, Romieu I, John EM. Racial and ethnic disparities in the impact of obesity on breast Cancer risk and survival: a global perspective. Adv Nutr. 2015;6(6):803-19.

35. Amadou A, Ferrari P, Muwonge R, Moskal A, Biessy C, Romieu I, et al. Overweight, obesity and risk of premenopausal breast cancer according to ethnicity: a systematic review and dose-response meta-analysis. Obes Rev. 2013;14(8):665-78.

36. Clarke CA, Keegan THM, Yang J, Press DJ, Kurian AW, Patel AH, et al. Agespecific incidence of breast Cancer subtypes: understanding the blackwhite crossover. J Natl Cancer Inst. 2012;104(14):1094-101. 
37. Horne HN, Beena Devi CR, Sung H, Tang TS, Rosenberg PS, Hewitt SM, et al. Greater absolute risk for all subtypes of breast cancer in the US than Malaysia. Breast Cancer Res Treat. 2015;149(1):285-91.

38. Juret P, Couette JE, Mandard AM, Carre A, Delozier T, Brune D, et al. Age at menarche as a prognostic factor in human breast cancer. Eur J Cancer. 1976;12(9):701-4.

39. Pike MC, Krailo MD, Henderson BE, Casagrande JT, Hoel DG. Hormonal risk factors, breast tissue age and the age-incidence of breast cancer. Nature. 1983;303(5920):767-70

40. Colditz GA. Relationship between estrogen levels, use of hormone replacement therapy, and breast Cancer. J Natl Cancer Inst. 1998;90(11):814-23.

41. Althuis MD, Fergenbaum JH, Garcia-Closas M, Brinton LA, Madigan MP, Sherman ME. Etiology of hormone receptor-defined breast Cancer: a systematic review of the literature. Cancer Epidemiol Biomarkers Prev. 2004; 13(10):1558-68.

42. Alsaker MDK, Opdahl S, Asvold BO, Romundstad PR, Vatten LJ. The association of reproductive factors and breastfeeding with long term survival from breast cancer. Breast Cancer Res Treat. 2011;130(1):175-82.

43. Urruticoechea A, Smith IE, Dowsett M. Proliferation marker Ki-67 in early breast cancer. J Clin Oncol. 2005;23(28):7212-20.

44. Reed A, Kutasovic J, Lakhani S, Simpson P. Invasive lobular carcinoma of the breast: morphology, biomarkers and 'omics. Breast Cancer Res. 2015;17(1):12.

45. Arpino G, Bardou V, Clark G, Elledge R. Infiltrating lobular carcinoma of the breast: tumor characteristics and clinical outcome. Breast Cancer Res. 2004; 6(3):R149-R56.

46. Chlebowski RT, Aiello E, McTiernan A. Weight loss in breast Cancer patient management. J Clin Oncol. 2002;20(4):1128-43.

47. Chan DSM, Vieira AR, Aune D, Bandera EV, Greenwood DC, McTiernan A, et al. Body mass index and survival in women with breast cancer-systematic literature review and meta-analysis of 82 follow-up studies. Ann Oncol. 2014;25(10):1901-14

48. Nechuta S, Chen WY, Cai H, Poole EM, Kwan ML, Flatt SW, et al. A pooled analysis of post-diagnosis lifestyle factors in association with late estrogenreceptor-positive breast cancer prognosis. Int J Cancer. 2016;138(9):2088-97.

49. Zhang M, Cai H, Bao P, Xu W, Qin G, Shu XO, et al. Body mass index, waistto-hip ratio and late outcomes: a report from the shanghai breast Cancer survival study. Sci Rep. 2017;7(1):6996.

50. Moon H-G, Han W, Noh D-Y. Underweight and breast Cancer recurrence and death: a report from the Korean breast Cancer society. J Clin Oncol. 2009;27(35):5899-905.

51. Kawai M, Minami Y, Nishino Y, Fukamachi K, Ohuchi N, Kakugawa Y. Body mass index and survival after breast cancer diagnosis in Japanese women. BMC Cancer. 2012;12(1):149.

52. Kawai M, Tomotaki A, Miyata H, Iwamoto T, Niikura N, Anan K, et al. Body mass index and survival after diagnosis of invasive breast cancer: a study based on the Japanese National Clinical Database—Breast Cancer Registry. Cancer Med. 2016;5(6):1328-40

53. Iyengar NM, Hudis CA, Dannenberg AJ. Obesity and inflammation: new insights into breast Cancer development and progression. Am Soc Clin Oncol Educ Book. 2013:33:46-51.

54. Thomas F, Bean K, Pannier B, Oppert J-M, Guize L, Benetos A. Cardiovascular mortality in overweight subjects. The Key Role of Associated Risk Factors. 2005;46(4):654-9.

Ready to submit your research? Choose BMC and benefit from:

- fast, convenient online submission

- thorough peer review by experienced researchers in your field

- rapid publication on acceptance

- support for research data, including large and complex data types

- gold Open Access which fosters wider collaboration and increased citations

- maximum visibility for your research: over $100 \mathrm{M}$ website views per year

At BMC, research is always in progress.

Learn more biomedcentral.com/submissions 